\title{
Untersuchungen über eine teilmechanisierte Glucose-Dehydrogenase-Mikromethode zur Bestimmung der Glukosekonzentration in nicht enteiweißtem Vollblut und Serum
}

\author{
U. Eßinger \\ Institut für Labormedizin, Städtische Kliniken, 6100 Darmstadt
}

\section{Zusammenfassung:}

Eine Adaption der Glucose-Dehydrogenase-Methode an eine LKB-Gerätekombination, bestehend aus Reaction Rate Analyzer 2086, 1-Channel Potentiometer Recorder 2066 und Kinetic Data Processor 2082 wird beschrieben. Die Bestimmung der Blutzuckerkonzentration erfolgt aus Hämolysat von $50 \mu \mathrm{l}$ Vollblut oder aus $50 \mu /$ Serum; eine Enteiweißung ist nicht erforderlich. Die vorgestellte Methode bietet die Möglichkeit, in einer Stunde bis zu 124 Blutzuckerbestimmungen in einem Meßbereich zwischen 20 und $1000 \mathrm{mg} / \mathrm{dl}$ durchzuführen.

Die Richtigkeit des Verfahrens wird durch Messen wäßriger und eiweißhaltiger Kontrollproben überprüft. Ein Vergleich der Methode mit der Neocuproin-Methode am AutoAna/yzer II und der HexokinaseMethode am ACA ergibt gute Korrelationskoeffizienten ( $r=0,994$ bis 0,998).

\section{Schlüsse/wörter:}

Blutglukosebestimmung - Teilmechanisierte Methode - Glucose-Dehydrogenase-Methode - LKBReaction Rate Instrument - Präzision und Richtigkeit.

\section{Summary:}

Adaption of the glucose dehydrogenase method to an $\angle K B$ equipment set-up, consisting of Reaction Rate Analyzer 2086, 1-Channel Potentiometer Recorder 2066. and Kinetic Data Processor 2082, is described. The blood sugar concentration is determined from the haemolysate of $50 \mu /$ of whole blood or from $50 \mu /$ of serum, deproteinization is not required. The method offers the possibility of performing up to 124 determinations of blood sugar within a measuring range between 20 and $1000 \mathrm{mg} / \mathrm{dl}$ within one hour. The accuracy is proved by checking aqueous and protein containing control samples. A comparison with the Technicon neocuproine method and the ACA hexokinase method shows good correlation coefficients $(r=0,994-0,998)$.

\section{Key words:}

Blood glucose determinations - Partly mechanical method - LKB-Reaction Rate Instrument - Precision and accuracy.

\section{Einleitung}

Im klinisch-chemischen Laboratorium ist Glukose neben Hämoglobin der mit Abstand am häufigsten bestimmte Parameter. Die Anwendung einer für großen Probendurchsatz geeigneten Methode ist daher notwendig. Außerdem müssen Einzelmessungen im Rahmen der Notfalldiagnostik ohne größere Zeitverluste für Geräteeichung und -vorbereitung möglich sein.
Heute werden meist enzymatische Blutzuckerbestimmungsmethoden angewandt. Unter diesen hat sich das Bestimmungsverfahren mittels Glucose-Dehydrogenase rasch einen Platz im klinisch-chemischen Labor erobert (1). Die bisher publizierten Methoden dieser Art verwenden als Untersuchungsmaterial chemisch oder durch Dialyse enteiweißtes Vollblut, Serum oder Plasma $(2-8)$. In beiden Fällen sind zusätzliche zeitund arbeitsaufwendige Schritte zur Probenvorberei- 
tung notwendig. An dieser Stelle soll erstmals über Erfahrungen mit einer Hämolysatmethode berichtet werden, die keine vorherige Enteiweißung der Probe notwendig macht.

\section{Material und Methoden}

Folgende Methoden zur Blutzuckerbestimmung wurden angewandt und miteinander verglichen:

Neocuproinmethode am AutoAnalyzer II" (9)

Hexokinasemethode am ACA (10)

Glucose-Dehydrogenase-Methode (1) in Adaptation an eine LKB-Gerätekombination:

\section{Reagenzien:}

System Glukose (Gluc-DH-Methode, UV-Test).

Enzymgemisch und Coenzym.

Merck: Art. Nr. 14055

System Glukose (Gluc-DH-Methode, UV-Test)

Pufferlösung

Merck: Art. Nr. 14051

Digitonin

Merck: Art. Nr. 3043

Maleinimid

Merck-Schuchardt: Art. Nr. 820754

Preciset Glucose

Boehringer Mannheim: Best. Nr. 125563.

Gebrauchslösungen:

Hämolysierlösung:

Digitoninstammlösung $(10 \mathrm{~g} / \mathrm{l})$

Maleinimid

Aqua bidest.

$10 \mathrm{ml}$

$100 \mathrm{mg}$

ad $1000 \mathrm{ml}$

Lösung 1 (Enzymgemisch) = Startreagenz:

Inhalt einer Flasche (1) „Enzymgemisch" mit $100 \mathrm{ml}$

Puffer lösen.

\section{Lösung 2 (Coenzym):}

Inhalt einer Flasche (2) "Coenzym" mit $500 \mathrm{ml}$ Puffer lösen.

Haltbarkeit der Lösungen bei $25^{\circ} \mathrm{C}$ ca. 2 Wochen, bei $4^{\circ} \mathrm{C}$ ca. 4 Wochen.

Konzentrationen im Ansatz:

\section{Gluc-DH}

Mutarotase

$4,000 \mathrm{kU} / \mathrm{I}$

$0,085 \mathrm{kU} / \mathrm{I}$

NAD

Phosphatpuffer pH 7,6

$0,846 \mathrm{mmol} / \mathrm{l}$

Natriumchlorid

Digitonin

Maleinimid
$0,185 \mathrm{~mol} / \mathrm{l}$

$0,231 \mathrm{~mol} / \mathrm{l}$

$0,006 \mathrm{mmol} / \mathrm{l}$

$0,073 \mathrm{mmol} / \mathrm{l}$

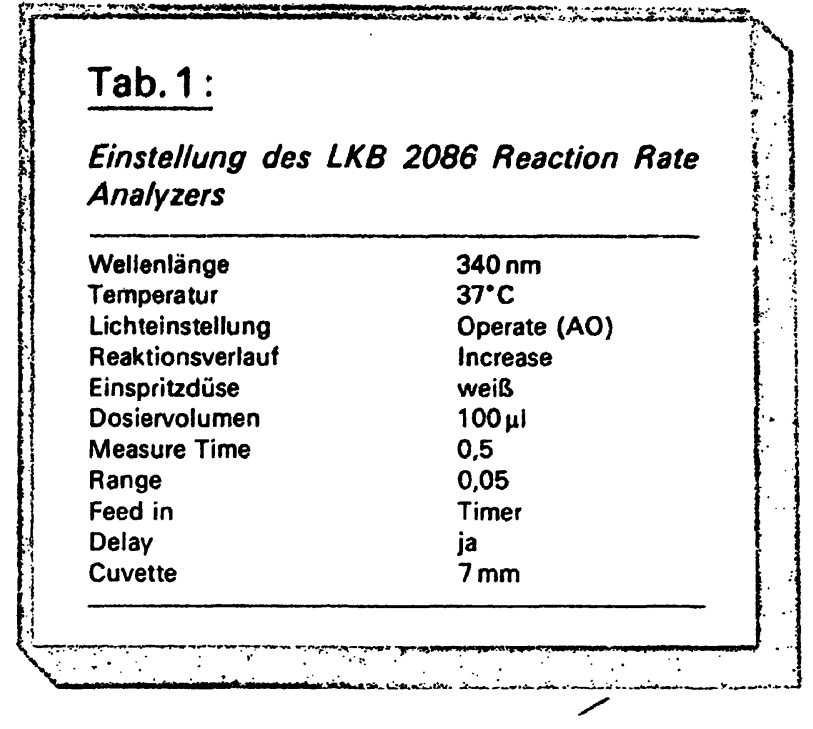

Eichstandards:

Zum Erstellen der Eichgeraden wurde Preciset Glucose, Boehringer Mannheim, Best. Nr. 125563, verwendet.

Geräte

Als Meßinstrument diente ein LKB Reaction Rate Analyzer in Verbindung mit einem LKB 2066 1-Channel Potentiometer Recorder und einem LKB 2082 Kinetic Data Processor. Geräteeinstellung siehe Tabelle 1.

Die Auswertung der Analysenergebnisse geschieht vollautomatisch unter Verwendung desLKB-Computerprogramms Fixed Time 2082-075 mit linearer Regression. Die Meßdauer beträgt $15 \mathrm{sec}$. Die Registrierung beginnt $5 \mathrm{sec}$ nach Zugabe des Startreagenzes. Die interne Eichgerade wird durch Messen von 3 Standardproben $(100,200$ und $400 \mathrm{mg} / \mathrm{dl})$ in Doppelbestimmung erstellt und bis zur Messung einer neuen Eichgeraden vom Rechner gespeichert. Wahlweise kann die Umrechnung der Photometermeßwerte in Konzentrationseinheiten auch durch Eingeben eines Faktors in den Data Processor erfolgen.

Zum Pipettieren der Hämolysierlösung und zum Weiterverdünnen des Hämolysates wurden 2 EppendorfProbe-Reagenzdosierer 5231 bzw. 5232 verwendet.

\section{Ausführung der Bestimmungen}

Die Probe (Kapillarblut, Venenblut ${ }^{1}$, Serum oder Eichstandard) wird mittels $50 \mu$ l End-zu-End Kapillarpipetten in $1 \mathrm{ml} \mathrm{Hämolysierlösung,} \mathrm{vorgelegt} \mathrm{in} 12 \times 55 \mathrm{~mm}$ Kunststoffröhrchen, überführt und durch mehrmaliges

\footnotetext{
Es wurden EDTA-Kalium und Jodacetat-Natrium enthaltende Probenröhrchen de Fa. W. Sarstedt, 5223 Nümbrecht-Rommelsdorf, verwendet.
} 
Schütteln der mit passenden Kunststoffstopfen verschlossenen Röhrchen ausgespült. LKB-Küvettenmagazine 8650 werden mit $7 \mathrm{~mm}$ Rundküvetten 2174-091 gefüllt und diese mittels Probe-Reagenzdosierer mit $50 \mu \mathrm{l}$ Primärverdünnung und $500 \mu \mathrm{l}$ Lösung 2 beschickt. Küvetteninhalt durch Schütteln und $\mathrm{Be}$ klopfen der Küvetten von Luftblasen befreien. Nach Einschieben der Magazine in die Einfüllschiene des Reaction Rate Analyzers kann die Analysenserie gestartet werden. Die Eichung des Rechners sollte täglich zu Beginn der Routinemessungen oder zwischenzeitlich bei Verwendung eines neuen Ansatzes von Hämolysierlösung, Coenzym oder Enzymgemisch vorgenommen werden. Es empfiehlt sich, am Anfang eines jeden Küvettenmagazins eine Standardprobe zu analysieren. Wird die Messung zwischen zwei Analysenserien längere Zeit unterbrochen, so sollte vor Beginn der neuen Serie die Startreagenzpumpe kurz durchgespült werden.

\section{Ergebnisse}

\section{Richtigkeit}

Die Richtigkeit der Methode wurde mit 6 verschiedenen käuflichen Kontrollseren überprüft. Die hierbei erhaltenen Ergebnisse sind in Tabelle 2 zusammengefaßt.

\section{Tab. 2:}

Richtigkeitskontrolle mit käuflichen Kontrollseren. Vergleich mit den Sollwertangaben der Hersteller für die Hexokinase- und Glucose-Dehydrogenase-Methode in $\mathrm{mg} /$ $100 \mathrm{ml}$

\begin{tabular}{|c|c|c|c|c|}
\hline \multirow{2}{*}{$\begin{array}{l}\text { Kontroll- } \\
\text { serum }\end{array}$} & \multirow[t]{2}{*}{$\mathbf{n}$} & \multicolumn{2}{|c|}{ Sollwert } & \multirow{2}{*}{$\begin{array}{l}\text { Ergeb- } \\
\text { nis } \\
\text { Gluc- } \\
\text { DH }\end{array}$} \\
\hline & & $\begin{array}{l}\text { Hexo- } \\
\text { kinase }\end{array}$ & $\begin{array}{l}\text { Gluc- } \\
\text { DH }\end{array}$ & \\
\hline & & $\mathrm{mg} / \mathrm{dl}$ & $\mathrm{mg} / \mathrm{dl}$ & $\mathrm{mg} / \mathrm{dl}$ \\
\hline $\begin{array}{l}\text { Monitrol II } \\
51 \mathrm{~B}\end{array}$ & 20 & 228 & 222 & 228 \\
\hline $\begin{array}{l}\text { Labtrol } \\
52 \mathrm{~A}\end{array}$ & 5 & 115 & 109 & 115 \\
\hline $\begin{array}{l}\text { Fluinorm } \\
1601 \mathrm{E}\end{array}$ & 5 & 116 & 112 & 117 \\
\hline $\begin{array}{l}\text { Precinorm U } \\
713\end{array}$ & 5 & 108 & 99 & 110 \\
\hline $\begin{array}{l}\text { Precilip } \\
456 \mathrm{~B}\end{array}$ & 5 & 103 & - & 103 \\
\hline $\begin{array}{l}\text { Seronorm } \\
130\end{array}$ & 20 & 115 & - & 118 \\
\hline
\end{tabular}

\begin{tabular}{|c|c|c|c|c|}
\hline \multicolumn{5}{|c|}{ Präzision in der Serie und von Tag zu Tag } \\
\hline & $\mathbf{n}$ & $\begin{array}{l}\bar{x} \\
\mathbf{m g} / \mathrm{dl}\end{array}$ & $\begin{array}{l}\mathrm{s} \\
\mathrm{mg} / \mathrm{dl}\end{array}$ & $\begin{array}{l}\text { VK } \\
\%\end{array}$ \\
\hline $\begin{array}{l}\text { In der } \\
\text { Serie }\end{array}$ & $\begin{array}{l}20 \\
20 \\
20\end{array}$ & $\begin{array}{r}234 \\
118 \\
52\end{array}$ & $\begin{array}{l}3,66 \\
2,58 \\
1,77\end{array}$ & $\begin{array}{l}1,56 \\
2,19 \\
3,41\end{array}$ \\
\hline $\begin{array}{l}\text { Von Tag } \\
\text { zu Tag }\end{array}$ & $\begin{array}{l}20 \\
20\end{array}$ & $\begin{array}{l}228 \\
109\end{array}$ & $\begin{array}{l}3,74 \\
3,94\end{array}$ & $\begin{array}{l}1,64 \\
3,63\end{array}$ \\
\hline
\end{tabular}

\section{Präzision}

Die Präzision in der Serie und von Tag zu Tag für 3 bzw. 2 verschiedene Glukosekonzentrationen ist in Tabelle 3 aufgeführt.

\section{Linearität}

Die Überprüfung der Linearität erfolgte mit wäßrigen Standards für einen Konzentrationsbereich von 10$1000 \mathrm{mg} / \mathrm{dl}$ und mit Jodacetatblut für einen Konzentra-

\section{Abb. 1:}

Prüfung der Linearität im Bereich von 0,555-55,5 mmol/l (10-1000 $\mathrm{mg} /$ $100 \mathrm{ml}$ ) mit wäßrigen Standardlösungen unter Verwendung von Preciset-Glucose (Boehringer Mannheim)






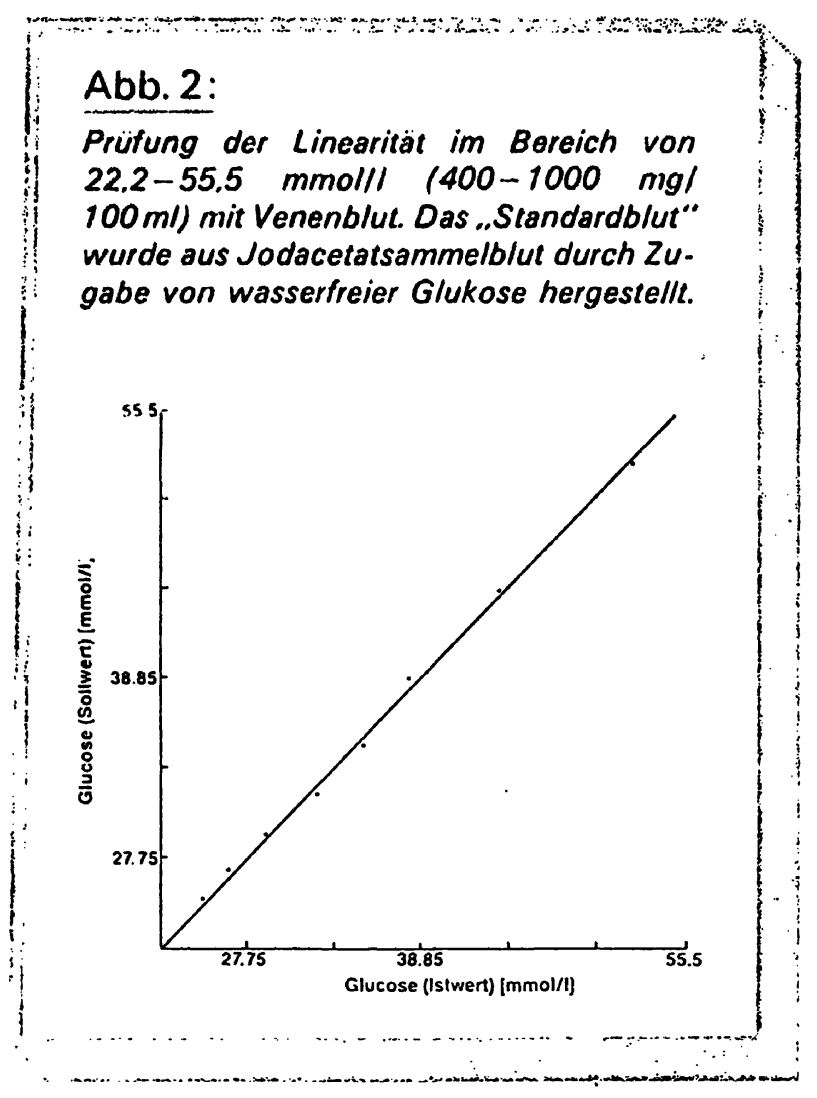

tionsbereich von $400-1000 \mathrm{mg} / \mathrm{dl}$. Wie die Abbildungen 1 und 2 zeigen, ist in beiden Fällen eine befriedigende Linearität über den untersuchten Konzentrationsbereich gegeben.

\section{Methodenvergleich}

Die Glucose-Dehydrogenase-Methode wurde an 101 Patientenseren mit der Hexokinase-Methode am ACA und an 100 Kapillar- und Jodacetatblutproben mit der Neocuproin-Methode am AutoAnalyzer II verglichen. Die Ergebnisse sind in den Abbildungen 3 bis 5 graphisch und statistisch dargestellt.

\section{Diskussion}

Die Glucose-Dehydrogenase besitzt praktisch die gleiche Spezifität wie die Hexokinase (11). Sie erlaubt jedoch die direkte photometrische Erfassung der Glukose ohne Indikatorreaktion, so daß Störeinflüsse bedingt durch Hilfsreaktionen nicht zu befürchten sind. Große Haltbarkeit und günstiger Preis der Reagenzien sind weitere Vorzüge der auf diesem Enzym basierenden Blutzuckerbestimmungsmethoden.

Darüber hinaus bietet die hier gewählte Versuchsanordnung und Gerätekombination eine Reihe zusätzlicher Vorteile. Die kurze Meßdauer gestattet bei einer für die

\section{Abb. 3:}

Glucosebestimmung in Kapillarblutproben. Vergleich zwischen der NeocuproinAutoAnalyzer I-Methode ( $x$ ) und der Glucose-Dehydrogenase-Methode (y). $N=100 ; y=-0,85+1,006 x ; r=0,997$ : $x=164,57 ; y=164,71 ; s_{y \cdot x}= \pm 7,24$. $27.75 \mathrm{mmol} / \mathrm{l}$ Glucose $=500 \mathrm{mg} / 100 \mathrm{ml}$

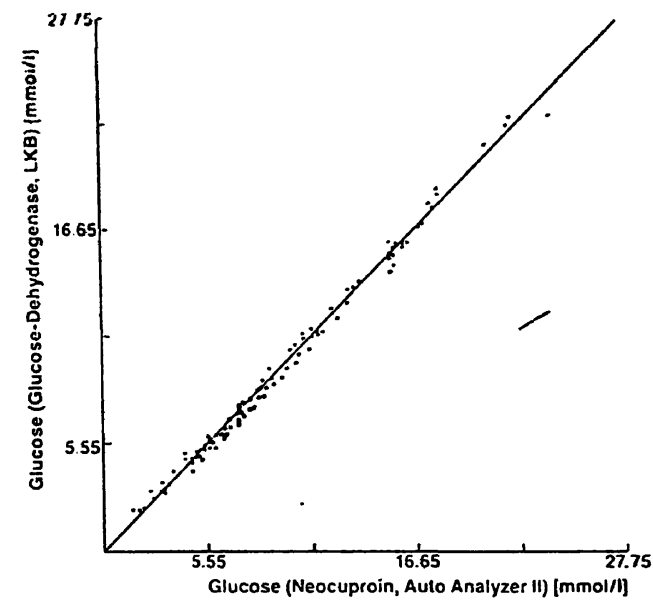

\section{Abb. 4:}

Glucosebestimmung in Jodacetatblutproben. Vergleich zwischen der NeocuproinAutoAnalyzer II-Methode $(x)$ und der Glucose-Dehydrogenase-Methode (v). $N=100 ; y=-4,12+1.051 x ; r=0,994$; $x=116,74 ; \quad y=118,57 ; s_{y \cdot x}= \pm 6,39$. $27,75 \mathrm{mmol} / / \mathrm{Glucose}=500 \mathrm{mg} / 100 \mathrm{ml}$






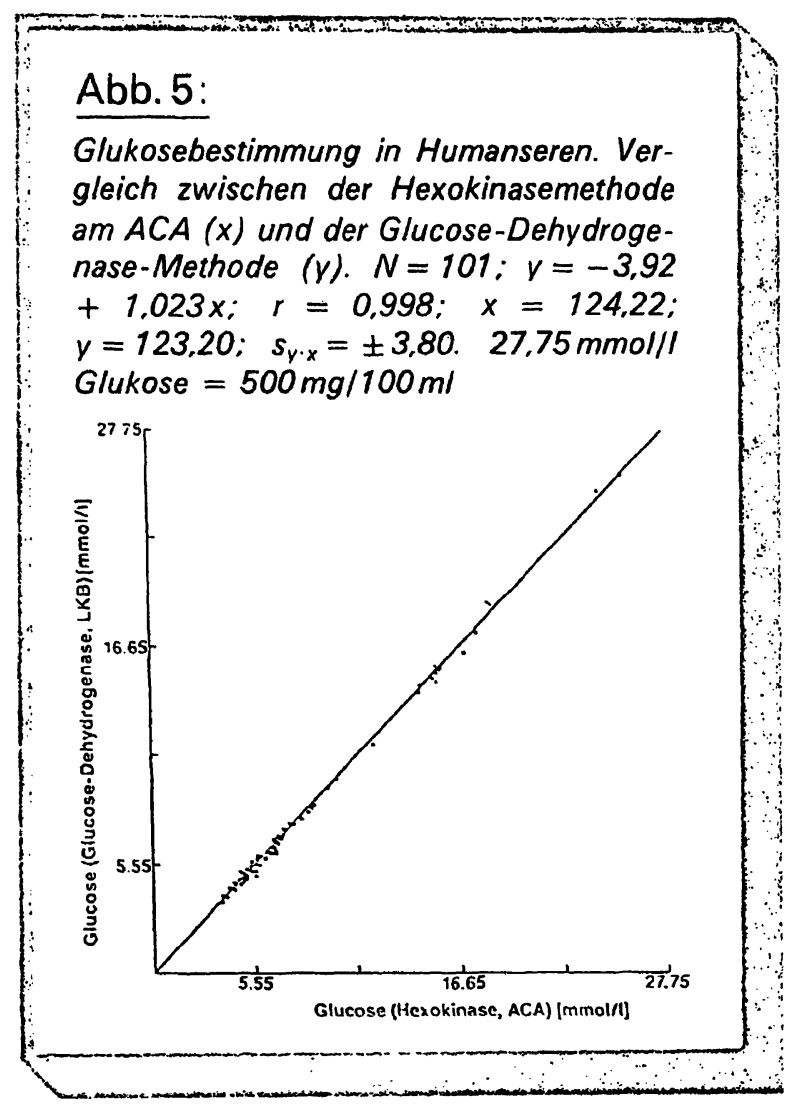

tägliche Routine akzeptablen Präzision die Analyse von 124 Proben pro Stunde. Die Methode ist bis $1000 \mathrm{mg} / \mathrm{dl}$ linear, so daß eine zusätzliche Probenverdünnung bei hohen Glukosekonzentrationen fast ausnahmslos entfällt. Eine Enteiweißung des Untersuchungsmaterials ist nicht notwendig. Hieraus resultiert eine nicht unerhebliche Zeit- und Arbeitsersparnis. Das Meßgerät kann Änderungen der Hintergrundsextinktion bis zu 1,1 E automatisch ausgleichen. Dieser Kompensationsbereich kann durch Veränderung der Lampenspannung innerhalb des vom Gerät gedeckten Extinktionsbereiches von 0 bis $1,8 \mathrm{E}$ beliebig verschoben werden. So ist es möglich sowohl wäßrige Standards und Seren als auch Kapillar- und Venenblutproben bis zu einer Hämoglobinkonzentration von $20 \mathrm{~g} / \mathrm{dl}$ mit derselben in Tabelle 1 angegebenen Lichteinstellung zu messen. Wie Tabelle 4 zeigt, haben Hämoglobinkonzentrationen von 5 bis $20 \mathrm{~g} / \mathrm{dl}$ keinen signifikanten Einfluß auf die Meßergebnisse $(0,9>p$ $>0,8$ ). Bei Hämoglobinwerten über $20 \mathrm{~g} / \mathrm{dl}$ kann das Meßgerät die Hintergrundextinktion gelegentlich nicht mehr kompensieren, es erfolgt der Ausdruck ,dark" am Rechner. In diesen Fällen kann die Messung bei leicht veränderter Lichteinstellung (Verdrehen des entsprechenden Regelknopfes um ca. $90^{\circ}$ im Uhrzeigersinn) durchgeführt werden. Experimentelle Untersuchungen haben gezeigt, daß die Veränderung der Lichteinstellung in dem in Frage kommenden Bereich entsprechend

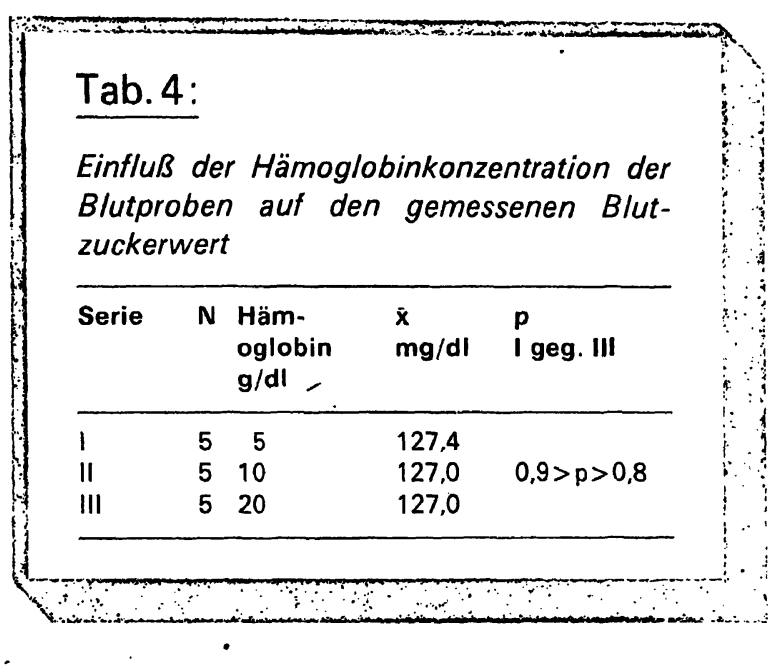

einer Änderung der Schreiberanzeige um $60 \mathrm{mV}$ ohne signifikanten Einfluß auf die Meßergebnisse bleibt $(0,2>p>0,1)$.

Durch die geräteinterne Speicherung der in der Regel einmal täglich einzugebenden Eichgeraden können im Nacht- und Notfalldienst jederzeit Einzelblutzuckerbestimmungen ohne vorherige nochmalige Geräteeichung durchgeführt werden. Während des Vorwärmund Meßvorgangs ist das med.-technische Personal für andere Arbeiten frei.

Schrifttum:

1. BANAUCH, D., BRÜMMER, W., EBELING, W., METZ, H., RINDFREY, H., LANG, H.: Eine Glucose-Dehydrogenase für die Glucose-Bestimmung in Körperflüssigkeiten. Z. Klin. Chem. Klin. Biochem. 13, 101 (1975).

2. RINDFREY, H., HELGER, R., LANG, H.: Kinetic determination of glucose concentrations with glucose dehydrogenase. Z. Klin. Chem. Klin. Biochem. 15, 217 (1977).

3. KELLER, H., WOLF, V.: Ein Verfahren zur enzymkinetischen Bestimmung von Glucose. Z. Klin. Chem. Klin. Biochem. 14, 27 (1976).

4. SCHOLER, A., PIANEZZI, A.: Mikromethode zur Bestimmung der GlucoseKonzentration mit Glucose-Dehydrogenase auf dem AutoAnalyser. Z. Klin. Chem. Klin. Biochem. 14, 189 (1976).

5. KORNMÜLLER, K. J., MÜLLER-PLATHE, O.: Reagenziensparende Modifikation der Glucose-Dehydrogenase-Methode für den AutoAnalyzer II zur Glucosebestimmung in Venen- und Kapillarblut. Z. Klin. Chem. Klin. Biochem. 15, 603 (1977).

6. MÜLLER, H. A. G.: Eine Glucosebestimmung aus Kapillarblut mit der GlucoseDehydrogenase-Methode am AutoAnalyzer I!. Lab. med. 2, 88 (1978).

7. LUTZ, R. A. FLÜCKIGER, J.: Kinetic determination of glucose with the GEMSAEC (ENI) centrifugal analyzer by the glucose dehydrogenase reaction, and comparison with two commonly used procedures. Clin. Chem. 21, 1372 (1975).

8. MÜLLER-MATTHESIUS, R.: Enzymkinetische Glucosebestimmung nach de Glucose-Dehydrogenase-Methode. Z. Klin. Chem. Klin. Biochem. 13, 187 (1975).

9. AutoAnalyzer Bulletin 2032-11-70-2. Technicon (1970).

10. ACA Chemistry Instruction Manual.

11. GERBIG, K.: Eine neve hochspezifische und praktikable Methode zur GlucoseBestimmung mit Glucose-Dehydrogenase. Med. Labor. 29, 1 (1976).

Anschrift des Verfassers:

Dr. med. Udo EBinger

Institut für Labormedizin

Städtische Kliniken

6100 Darmstadt

Grafenstraße 9 